\title{
Extracting Personal Information from Conversations
}

\author{
Anna Tigunova \\ Max Planck Institute for Informatics \\ Saarland Informatics Campus, Saarbrücken, Germany \\ tigunova@mpi-inf.mpg.de
}

\begin{abstract}
In my doctoral research, I focus on inferring personal information from conversational texts. Such unstructured input does not provide sufficient explicit assertions, hence, personal facts have to be inferred from latent cues or speech style. In my work I investigate what salient attributes can be extracted from dialogues and which tools may be applicable for that.
\end{abstract}

\section{CCS CONCEPTS}

- Computing methodologies $\rightarrow$ Information extraction.

\section{KEYWORDS}

Information extraction; personal knowledge; user profiling; conversational text; neural networks

\section{ACM Reference Format:}

Anna Tigunova. 2020. Extracting Personal Information from Conversations. In Companion Proceedings of the Web Conference 2020 (WWW'20 Companion), April 20-24, 2020, Taipei, Taiwan. ACM, New York, NY, USA, 5 pages. https://doi.org/10.1145/3366424.3382089

\section{INTRODUCTION}

Motivation. Intelligent assistants have become an essential part of life, being able to deal with a wide range of routine tasks and provide factual information. Still, there is a growing demand for creating user-oriented chatbot agents, which are able to hold personalized conversations. Such chatbots should be aware of users' interests and background to produce meaningful or even funny responses. Consider the following conversation between a person $(\mathrm{P})$ and a chatbot (C):

$\mathrm{P}$ : It was a hard marathon for an armature, the sneakers became my second feet!

C: Hope you'll have a good rest! By the way, have you heard about the recent shocking research on protein absorption?

$\mathrm{P}$ : Interesting, no... Tell me more

In this conversation, knowing that the user has athletics as a hobby, the chatbot is able to suggest the discussion topic on sport nutrition, which may be interesting to the user. However, to avoid the necessity for the user to provide their information, the agent should be capable of learning the personal facts directly from their utterances.

This paper is published under the Creative Commons Attribution 4.0 International (CC-BY 4.0) license. Authors reserve their rights to disseminate the work on their personal and corporate Web sites with the appropriate attribution.

WWW'20 Companion, April 20-24, 2020, Taipei, Taiwan

() 2020 IW3C2 (International World Wide Web Conference Committee), published under Creative Commons CC-BY 4.0 License.

ACM ISBN 978-1-4503-7024-0/20/04.

https://doi.org/10.1145/3366424.3382089
In our example, the chatbot should have used the words "marathon" and "sneakers" to infer the knowledge about the user's hobby.

Thus, we are interested in automated methods to learn personal facts from dialogues. This arouses the following issues:

- What to extract: which personal facts would be feasible to infer from conversations, which would be useful for the applications

- How to extract: which tools would be suitable regarding the properties of conversational texts

- How to use: what are the potential applications for the acquired knowledge and how to incorporate it

Concerning the first issue, we define the personal facts to be attributes (age or profession), interests, skills, relationships with other people (family or business relations), sentiments and personality.

The second issue can not be directly addressed by existing Information Extraction methods. An extensive prior work on IE generally focuses on formal texts, such as news articles and Wikipedia pages. The colloquial nature of dialogues provides an additional challenge: the utterances are short and noisy [35], there are often topic drifts [8] and a lot of pronouns, which require thorough coreference resolution [10]. Nevertheless, conversations are a rich source of descriptive and emotional signal, hence, they are well suited for inferring personal traits.

On the application side we identify the possible uses as enhanced chatbots, personalized recommendations and search. The extracted facts can form a personal knowledge base, a reusable asset to manage and transfer personal data $[3,35]$.

Limitations of the State of the Art. The previous work has mostly been focusing on capturing personal attributes from explicit mentions [16, 35], which are, however, scarce in real conversations. The attempts to learn the features implicitly have been restricted to the attributes having a small number of values, such as gender or age $[22,24]$.

On the other hand, there has been a significant research effort on creating a latent representation of speaker [15, 17]. Such representation can be directly used in a dialogue system [37]. Yet, such information is not scrutable, providing no possibility for the speaker to view and change it. Additionally, latent representations are difficult to transfer between models and applications.

Problem Statement. As a core topic of my dissertation, I pose the problem of predicting personal attributes from conversational data. In the current report I highlight our work on information extraction from dialogues, describe the developed approaches and provide an outlook, how we can further enhance our research.

\section{RELATED WORK}

Personal Information from Transcribed Speech. Our research concerns the textual representation of conversations, i.e. transcribed 
dialogues. They range from conversations between people to userchatbot interactions, which could be open-domain [15, 36] or taskoriented [11, 18, 23]. Another distinguishable dialogue source is literary plays and film scripts [17].

Prior work investigates the extraction of personal facts, which vary from gender $[8,16,33]$, age $[8,33]$ or ethnicity $[8,16]$, biography facts [10] to user preferences [18]. Furthermore, the speakers' relationships are often predicted, either as the polarity of the attitude towards the interlocutor [2] or a fine-grained relationship type [2,33].

Most prior work constructs a speaker's latent representation using linguistic feature sets [8, 10, 17], language models [30] or embeddings $[15,18]$. The disadvantage of creating such latent personality is its limited interpretability, preventing from checking its consistency with explicit attributes.

Few research efforts are dedicated to distilling precise personal facts $[8,16,33]$, often requiring the search for explicit pattern mentions ("I am a doctor") [16]. However, such assertions are rare in real conversations, yielding a poor recall of the models. Instead, some authors resort to classification $[8,17]$ via linguistic features. Additionally, Garera and Yarowsky incorporate partner identity and $\mathrm{n}$-grams to classify age or gender using Support Vector Machines (SVMs) [8]. A major drawback of their work is that the inferred attributes take only binary values.

To predict explicit or latent personality, standard machine learning tools are commonly used, such as Logistic Regression [2] or Conditional Random Fields [15], which often operate on hand-crafted linguistic features [8]. Some works exploit conversational partner information, including their identity [2], personal traits [8] or their utterances [33]. More recently, the speaker's representations are built using neural approaches [11, 18, 33, 36].

Extracting Personal Information from Social Media. The texts stemming from social media are often short and colloquial, making them similar to spoken utterances. Moreover, the discussion threads in topical forums resemble the flow of a natural conversation. Most of existing work on user profiling focuses on Twitter $[12,13,15,21,22,24,28,35]$. Other research explores Facebook $[19,28]$, Reddit [9] and other platforms [37].

There has been significant effort on predicting age and gender of social media users $[13,24,28,37]$ and their personality $[9,19]$. Less explored but more specific attributes like origin [24], political views [21, 24], ethnicity [21], occupation [22] or mental health issues [29] are also considered. The representations utilized for social media profiling are built via linguistic features $[9,19]$, language models [28], n-grams [24] or embeddings [22]. These features are used afterwards in SVMs [9, 24], topical models [21] and neural networks $[13,37]$.

A common limitation of most prior work is the small number of attribute values to be predicted. More interesting are the traits with large or even open-ended value lists, such as interests $[1,12]$ or locations [14]. Their values are usually captured by detecting explicit mentions of entities and mapping them to Wikipedia concepts $[1,14]$.

Nevertheless, social media content is still different from natural conversational text, due to the additional signals (user activity or hashtags), which are often used alongside the texts $[9,24]$.

\section{PROPOSED APPROACH}

The most straightforward way to gather personal knowledge from conversations is to extract the triples <person, attribute,value>, which can be directly inserted into a personal knowledge base. This is not practical in the conversation domain, where the personal assertions are rarely explicitly expressed. Thus, we suggest viewing this problem as a classification task, where the attribute is known in advance and the goal is to predict its implicit value.

As seen in Section 2, there is a wealth of former work on predicting such speaker characteristics as gender or age, often posed merely as a binary classification task [13, 24, 28]. We find it intriguing to explore the personal attributes with very long or open-ended list of values, such as hobbies, which are more useful for downstream applications (personalized conversational agents and recommendations).

To address this task we propose two novel architectures, Hidden Attribute Models (HAMs) [32] and Retrieval-based Hidden Attribute Models (RHAM), tailored specifically towards inferring implicit personal traits from conversations. These models leverage latent textual cues to predict a value for the given attribute; in current work we investigate multi-value hobby and profession attributes. I provide the models' descriptions and the obtained experimental results in Sections 4.1 and 4.2.

However, one shortcoming of our models is that they fail to exploit the information coming from the dialogue partner. The conversation is essentially an interaction among multiple speakers, thus, the utterances of the interlocutor provide a vital context and can also act as a source of attribute cues. Considering this, we envision to extend our work by incorporating the texts from all conversation participants. A tangible methodology for that I propose in Section 4.3.

\section{METHODOLOGY}

\subsection{Hidden Attribute Models}

Architecture Description. Conversational data contains a lot of noise, many words (like interjections) and the whole utterances provide no valuable information [35, 37]. Moreover, the utterances, considered outside of their context, are useless.

Following these observations we propose Hidden Attribute Models (HAMs). Given a set of input utterances $U$, each one consisting of terms $t_{1}, \ldots, t_{M}$, HAMs work in three steps:

(1) Create a representation of the utterance, considering the importance of terms: $R_{i}^{u t t}=f_{u t t}\left(t_{1}, t_{2}, \ldots, t_{M}\right)$

(2) Create a representation of the speaker from all their utterances, considering their importance: $R^{s p}=f_{s p}\left(R_{1}^{u t t}, \ldots, R_{N}^{u t t}\right)$

(3) Given the speaker's representation, score the attribute values: $a_{k}=f_{o b j}\left(R^{s p}\right)$

$R_{i}^{u t t}$ and $R^{s p}$ are represented by attention mechanisms, which assign importance to the components of both speaker and utterance representations. As prediction HAM returns the value with the highest score $a_{k}$ from the fully-connected layer acting as $f_{o b j}$.

More details on HAM can be found in our paper [32].

Discussion. The advantage of HAM is that it is a light-weight model, considering the parameter number, required amount of training time and data. However, HAM's predictions are restricted 


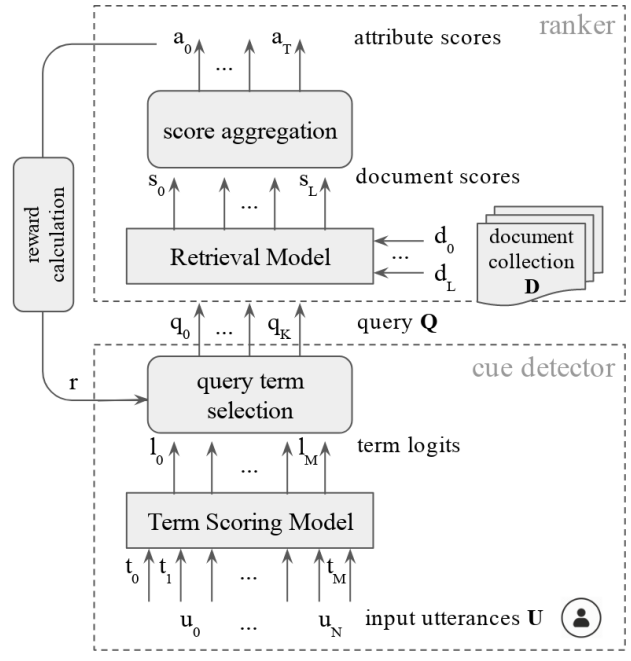

Figure 1: The pipeline of RHAM.

to a short list of values, which do not cover all possibilities for profession and hobby attributes. To be able to handle unlimited value lists we introduce a new retrieval-based model.

\subsection{Ongoing Work: Retrieval-based Hidden Attribute Models}

Overview. The bottleneck of supervised classification of numerous classes is the difficulty of finding sufficient training data for all values. Exploiting the assumption that the knowledge about the missing classes can be derived from external sources, we introduce RHAMs, Retrieval-based Hidden Attribute Models. RHAMs predict the attribute values that were not seen during training by using an external document collection. Assuming that this collection has all existing values, RHAMs can be considered to be applicable to the open-ended attributes.

Given the speaker's utterances as input, RHAMs identify the textual cues, which are used to query an external document collection. The Figure 1 shows the two core components of RHAMs, cue detection and document ranking.

Cue Detection. Let $t_{0} \ldots t_{M}$ be a concatenation of all terms in the input utterances. In the first stage the term scoring model assigns each term a score $l_{0} \ldots l_{M}$, indicating, the relevance of the term to the correct attribute value. Based on the assigned values, the top scoring $K$ terms are selected to form a query to the document ranking component. As the term selection process involves nondifferentiable argmax operation, we had to resort to use a policy gradient REINFORCE algorithm [31] to train our model.

The term scoring model can be implemented by any neural architecture, still a preference should be given to the one, which considers the context of the terms. First, the selection of terms should be guided by the surrounding context to avoid overfitting to the cues of the values observed during training. Second, the context helps to understand that the term is relevant to the given speaker. With this in mind, we used the BERT model [7] for selecting the terms because of its superior performance on many NLP tasks.
Document Ranking. In the second stage, the words, selected after cue detection, form a query $Q=q_{0} \ldots q_{K}$, which is used to rank the documents in the external collection $D=d_{0} \ldots d_{L}$. We assume that each document in the collection can be automatically associated with some attribute value. For example, a Wikipedia page "church" can be labeled with "priest" and "monk".

The ranker produces the scores $s_{0} \ldots s_{L}$, which denote the relevance of the query $Q$ to each of the documents in the collection. The scores for single documents may need to be aggregated, as each attribute value can be represented by several documents.After aggregation we obtain the final value scores $a_{0} \ldots a_{T}$, which we sort and return the top value as the prediction of RHAM.

The ranker can also be instantiated with any retrieval model. We utilized KNRM [34], a state of the art embedding-based neural architecture, which captures semantic term similarities via word embeddings.

Training. We applied REINFORCE algorithm to train our model in a semi-supervised way. Without the knowledge of the optimal query terms we could not calculate the loss for the term scoring model directly. REINFORCE helped to backpropagate through the parameters of this model,regardless of non-differentiable argmax function in the term selection stage.

We view such training as a policy gradient exploration process, where after sampling each query term we get an immediate reward from the ranker, informing if the selection was suitable. Thus, term selector acts as a policy, which at the timestep $\tau$ samples each input term with the probability $p_{\tau}$.

\subsection{Future Work: Incorporating the Conversational Partner}

Our models can efficiently tackle the peculiarities of the conversational genre, such as noise (by focusing on salient words) and brevity (by considering the complete history of utterances), yet they ignore one essential dialog aspect, which is the speech of the interlocutor. Here I provide the ideas how to incorporate it.

First, the ambiguities in the current subject's speech can be avoided by considering it in context of the interlocutor's utterances. Consider the following conversation:

\section{A: I take photos all day long}

B: Isn't it a nice thing to do for a living?

Without B's reply, it is unclear, whether A is referring to a job photographer or a hobby photography.

Next, hidden cues can also be extracted from the sentences of the dialogue partner. For example, from the dialogue
A: Oh, I am tired
B: I suppose that's because you glued that ship the whole day

we could get the cues "glue" and "ship" from B's speech to predict "modelling" as A's hobby, which can not be inferred from A's utterances alone. This raises the problem of determining, which person the interlocutor is referring to.

Finally, the input from both dialogue participants is crucial to identify the speakers' relationship. The former work on relationship prediction mostly focused on characters in fictional narratives [6] and was limited to a small number of inferred relationships [33]. 


\begin{tabular}{|c|c|c|c|c|c|}
\hline \multirow{2}{*}{ Model } & \multirow{2}{*}{$\begin{array}{l}\text { Document } \\
\text { collection }\end{array}$} & \multicolumn{2}{|c|}{ profession } & \multicolumn{2}{|c|}{ hobby } \\
\hline & & MRR & nDCG & MRR & nDCG \\
\hline N-GrAM & - & .13 & .43 & .11 & .40 \\
\hline W2V-C & - & .09 & .39 & .08 & .32 \\
\hline $\mathrm{CNN}$ & - & .20 & .52 & .14 & .43 \\
\hline BERT & - & .50 & .68 & .35 & .55 \\
\hline HAM & - & .32 & .59 & .33 & .55 \\
\hline RHAM & Wiki $_{\text {category }}$ & .43 & .62 & .31 & .51 \\
\hline & Web search & .49 & .66 & .31 & .51 \\
\hline
\end{tabular}

Table 1: Results of HAM and RHAM on seen experiments.

Taking into consideration the significance of the conversational partner speech and the challenge of handling it, I propose to make it the principal direction for our future work.

\section{EXPERIMENTAL RESULTS}

\subsection{Experimental Data}

We investigate two personal attributes, profession and hobby. Their possible values we draw from comprehensive Wikipedia lists ${ }^{1}$.

Input Utterances. For our experiments we used social media texts as a proxy for conversational data. We gathered the submissions of Reddit users, disregarding fantasy and dating subforums. All users were required to have 10-50 posts, each one being 10-40 words long. We left only the posts with personal pronouns (except the $3 \mathrm{rd}$ person) as the ones likely to contain personal assertions.

We automatically annotated the selected users with hobby and profession values using Snorkel [25], which combines noisy heuristics to derive the final probabilistic labeling estimates. We used two labeling strategies: (i) explicit pattern search (for example, "I am a doctor") and (ii) value-specific lexicon count (a doctor would often say "hospital", "leukocytes", "prescription").

For each possible attribute value Snorkel provides a labeling probability score, based on the results of these two strategies over the given user's utterances. We sort the users by the probability of being positively labeled and take the top ones.

Document Collections. Based on the attribute value lists, we automatically created and labeled two document collections:

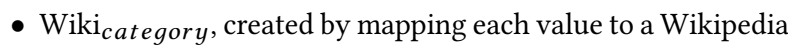
category and labeling each category subpage with this value

- Web search, comprising of 100 top results for querying a web search engine with queries "my job as a [profession value]" and "my favorite hobby is [hobby value]"

\subsection{Experimental Setup}

We evaluate our models in two settings. First, a seen setting, assuming that all attribute values from the test set were present during training. Second, an unseen case, in which the values for test and training are completely different. For the second case we use cross validation, splitting the set of attributes into 10 disjoint folds.

For the seen experimental setup we use the state-of-the art methods for users profiling: an SVM classifier (N-GrAM) [4], Gaussian Process classifier (W2V-C) [22] and convolutional neural network

${ }^{1}$ en.wikipedia.org/wiki/List_of_hobbies,

en.wikipedia.org/wiki/Lists_of_occupations

\begin{tabular}{|c|c|c|c|c|c|c|c|c|}
\hline \multirow{3}{*}{ Model } & \multicolumn{4}{|c|}{ profession } & \multicolumn{4}{|c|}{ hobby } \\
\hline & \multicolumn{2}{|c|}{ Wiki $_{\text {cat }}$} & \multicolumn{2}{|c|}{ Web search } & \multicolumn{2}{|c|}{ Wiki $_{\text {cat }}$} & \multicolumn{2}{|c|}{ Web search } \\
\hline & MRR & nDCG & MRR & nDCG & MRR & nDCG & MRR & nDCG \\
\hline RAKE & .19 & .39 & .11 & .28 & .14 & .37 & .07 & .23 \\
\hline TextRank & .26 & .45 & .15 & .32 & .20 & .42 & .10 & .28 \\
\hline RHAM & .35 & .55 & .41 & .59 & .27 & .49 & .19 & .38 \\
\hline
\end{tabular}

Table 2: RHAM performance results on unseen experiments compared to the baselines.

model [5]. In addition to that, we apply BERT, trained on classification task. For the unseen setup we select state of the art unsupervised keyword extraction methods, TextRank [20] and RAKE [27], which we pair with BM25 [26] for document ranking.

For quantitative evaluation we compute MRR (which we average across attribute values) and nDCG (which we average across users).

\subsection{Results}

Quantiative Results. We provide the evaluation of HAM only for the seen case and RHAM for both settings. The results of the seen experiment are presented in Table 1. We observe that both HAM and RHAM outperform author profiling baselines, each of them reaching a comparable performance with BERT.

The advantage of RHAM is that it can be used in the unseen case, as provided in Table 2. RHAM outperforms all unsupervised baselines on all document collections, which shows the benefit of training keyword detection in a joint pipeline.

Qualitative Results. We illustrate the word weighting mechanism of HAM in Figure 2 by providing the visualisation of attention weights for profession attribute. HAM puts greater attention weights on such words as "prosecution", "law", "guilty" to predict profession lawyer.

To show that distant supervision on ranked documents yields good keyword extraction, we aggregated the most prominent keywords for each attribute value, the results are provided in Table 3. We note that RHAM is able to select distinctive cues, such as "cake", which is more specific to baking than cooking. RHAM can also make sensible predictions for the long-tail values, such as model aircraft.

\begin{tabular}{lrlrlr}
\hline \multicolumn{2}{c}{$\begin{array}{c}\text { baking } \\
\text { (\#sample=64) }\end{array}$} & \multicolumn{2}{c}{$\begin{array}{c}\text { quilting } \\
\text { (\#sample=27) }\end{array}$} & \multicolumn{2}{c}{$\begin{array}{c}\text { model aircraft } \\
\text { (\#sample=2) }\end{array}$} \\
\hline cake & bread & sewing & way & cat & dimensions \\
food & cream & quilting & game & plane & pilots \\
recipe & cooking & quilt & metal & construction & song \\
cheese & pasta & fabric & design & planes & steam \\
baking & cook & music & playing & energy & music \\
\hline
\end{tabular}

Table 3: RHAM's top 10 terms per label for hobby attribute.

\section{DISCUSSION AND FUTURE WORK}

Extracting structured personal information is a crucial step towards personalizing chatbots, search and recommendations. Conversational data contains a wealth of such knowledge, which is, however, challenging to extract. The previous research attempts have mostly been limited to extraction from explicit mentions or classification of trivial attributes. Therefore, I find it a topical and intriguing issue to work upon. 


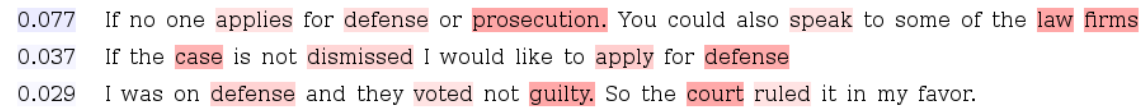

Figure 2: HAM attention visualisation for profession attribute

In this report I presented our current research, which partially tries to overcome the drawbacks of the previous systems. We developed HAM, a lightweight model for capturing textual cues efficiently, and RHAM, which is capable of predicting the values, absent from the training data. These models show promising results, quantitatively outperforming the baselines and giving qualitatively interpretable predictions. Additionally, I discussed my views on utilizing the utterances of conversational partner.

I summarize the general research directions for the future work, relevant to the questions posed in the introduction:

- What to extract Expanding the list of attributes with relationship between the speakers, favorite song/team/food or the speaker's personality

- How to extract Improving the existing models to exploit the speech of both dialog participants and generalize among different conversational datasets

- How to use Exploring the way the extracted personal information can be used in personalized chatbots or recommendations My research plan includes covering some of the above mentioned issues, as well as exploring other related tasks.

\section{ACKNOWLEDGMENTS}

I would like to thank my advisor, Professor Gerhard Weikum and my collaborators, Paramita Mirza and Andrew Yates for their supervision and encouragement.

\section{REFERENCES}

[1] Fabian Abel, Qi Gao, Geert-Jan Houben, and Ke Tao. 2011. Analyzing user modeling on twitter for personalized news recommendations. In ACM UMAP'11. Springer, 1-12

[2] Mahmoud Azab, Noriyuki Kojima, Jia Deng, and Rada Mihalcea. 2019. Representing Movie Characters in Dialogues. In Proceedings CoNLL'19. 99-109.

[3] Krisztian Balog and Tom Kenter. 2019. Personal Knowledge Graphs: A Research Agenda. In Proceedings of SIGIR'19. 217-220.

[4] Angelo Basile, Gareth Dwyer, Maria Medvedeva, Josine Rawee, Hessel Haagsma, and Malvina Nissim. 2017. N-GrAM: New Groningen Author-profiling ModelNotebook for PAN at CLEF 2017. In CLEF 2017 Evaluation Labs and WorkshopWorking Notes Papers. http://ceur-ws.org/Vol-1866/

[5] Roy Khristopher Bayot and Teresa Gonçalves. 2018. Age and Gender Classification of Tweets Using Convolutional Neural Networks. In Machine Learning, Optimization, and Big Data. Springer International Publishing, Cham, 337-348.

[6] Snigdha Chaturvedi, Mohit Iyyer, and Hal Daume III. 2017. Unsupervised learning of evolving relationships between literary characters. In Thirty-First AAAI Conference on Artificial Intelligence.

[7] Jacob Devlin, Ming-Wei Chang, Kenton Lee, and Kristina Toutanova. [n.d.] Bert: Pre-training of deep bidirectional transformers for language understanding. NAACL'19.

[8] Nikesh Garera and David Yarowsky. 2009. Modeling Latent Biographic Attributes in Conversational Genres. In Proceedings of ACL/IFCNLP'09.

[9] Matej Gjurković and Jan Šnajder. 2018. Reddit: A gold mine for personality prediction. In Proceedings of the 2nd Workshop on Comp. Modeling of People's Opinions, Personality, and Emotions in Social Media, NAACL-HLT'18. 87-97.

[10] Hongyan Jing, Nanda Kambhatla, and Salim Roukos. 2007. Extracting Social Networks and Biographical Facts From Conversational Speech Transcripts. In Proceedings of ACL'07.

[11] Chaitanya K. Joshi, Fei Mi, and Boi Faltings. 2017. Personalization in GoalOriented Dialog. In Proceedings of Conversational AI Workshop, NIPS'17.

[12] Pavan Kapanipathi, Prateek Jain, Chitra Venkataramani, and Amit Sheth. 2014 User interests identification on twitter using a hierarchical knowledge base. In
Proceedings of ESWC'14. Springer, 99-113.

[13] Sunghwan Mac Kim, Qiongkai Xu, Lizhen Qu, Stephen Wan, and Cecile Paris. 2017. Demographic Inference on Twitter using Recursive Neural Networks. In Proceedings of ACL'17.

[14] Revathy Krishnamurthy, Pavan Kapanipathi, Amit P Sheth, and Krishnaprasad Thirunarayan. 2014. Location prediction of twitter users using wikipedia. (2014).

[15] Jiwei Li, Michel Galley, Chris Brockett, Georgios P Spithourakis, Jianfeng Gao, and Bill Dolan. 2016. A persona-based neural conversation model. Proceedings of ACL'16.

[16] Xiang Li, Gökhan Tür, Dilek Z. Hakkani-Tür, and Qi Li. 2014. Personal knowledge graph population from user utterances in conversational understanding. In Proceedings of IEEE SLT'14.

[17] Grace I. Lin and Marilyn A. Walker. 2011. All the World's a Stage: Learning Character Models from Film. In Proceedings of AIIDE'11.

[18] Liangchen Luo, Wenhao Huang, Qi Zeng, Zaiqing Nie, and Xu Sun. 2019. Learning personalized end-to-end goal-oriented dialog. In Proceedings of the AAAI Conference on Artificial Intelligence, Vol. 33. 6794-6801.

[19] Dejan Markovikj, S. Gievska, Michal Kosinski, and David Stillwell. 2013. Mining Facebook Data for Predictive Personality Modeling. In ICWSM 2013.

[20] Rada Mihalcea and Paul Tarau. 2004. Textrank: Bringing order into text. In Proceedings of EMNLP'04. 404-411.

[21] Marco Pennacchiotti and Ana-Maria Popescu. 2011. A machine learning approach to twitter user classification. In Fifth International AAAI Conference on Weblogs and Social Media.

[22] Daniel Preoţiuc-Pietro, Vasileios Lampos, and Nikolaos Aletras. 2015. An analysis of the user occupational class through Twitter content. In Proceedings of ACL/IFCNLP'15 (Volume 1: Long Papers). 1754-1764.

[23] Kun Qian and Zhou Yu. 2019. Domain Adaptive Dialog Generation via Meta Learning. Proceedings of ACL'19.

[24] Delip Rao, David Yarowsky, Abhishek Shreevats, and Manaswi Gupta. 2010. Classifying latent user attributes in twitter. In Proceedings of the 2nd international workshop on Search and mining user-generated contents. ACM, 37-44.

[25] Alexander Ratner, Stephen H. Bach, Henry Ehrenberg, Jason Fries, Sen Wu, and Christopher Ré. 2017. Snorkel. Proceedings of the VLDB Endowment 11, 3 (Nov 2017), 269-282. https://doi.org/10.14778/3157794.3157797

[26] Stephen Robertson and Hugo Zaragoza. 2009. The Probabilistic Relevance Framework: BM25 and Beyond. Found. Trends Inf. Retr. 3, 4 (2009), 333-389.

[27] Stuart Rose, Dave Engel, Nick Cramer, and Wendy Cowley. 2010. Automatic keyword extraction from individual documents. Text mining: applications and theory 1 (2010), 1-20.

[28] Maarten Sap, Gregory Park, Johannes Eichstaedt, Margaret Kern, David Stillwell, Michal Kosinski, Lyle Ungar, and Hansen Andrew Schwartz. 2014. Developing Age and Gender Predictive Lexica over Social Media. In Proceedings EMNLP'14.

[29] Judy Hanwen Shen and Frank Rudzicz. 2017. Detecting anxiety through reddit. In Proceedings of the Fourth Workshop on Computational Linguistics and Clinical Psychology-From Linguistic Signal to Clinical Reality. 58-65.

[30] Yiping Song, Zequn Liu, Wei Bi, Rui Yan, and Ming Zhang. 2019. Learning to Customize Language Model for Generation-based dialog systems. arXiv preprint arXiv:1910.14326 (2019)

[31] Richard S Sutton, David A McAllester, Satinder P Singh, and Yishay Mansour. 2000. Policy gradient methods for reinforcement learning with function approximation. In Advances in neural information processing systems. 1057-1063.

[32] Anna Tigunova, Andrew Yates, Paramita Mirza, and Gerhard Weikum. 2019. Listening between the Lines: Learning Personal Attributes from Conversations. In Proceedings of WWW'19. 1818-1828.

[33] Charles Welch, Verónica Pérez-Rosas, Jonathan K. Kummerfeld, and Rada Mihalcea. 2019. Look Who's Talking: Inferring Speaker Attributes from Personal Longitudinal Dialog. In Proceedings of CICLing. Springer, La Rochelle, France. https://arxiv.org/abs/1904.11610

[34] Chenyan Xiong, Zhuyun Dai, Jamie Callan, Zhiyuan Liu, and Russell Power. 2017. End-to-End Neural Ad-hoc Ranking with Kernel Pooling. In Proceedings of SIGIR'17.

[35] An-Zi Yen, Hen-Hsen Huang, and Hsin-Hsi Chen. 2019. Personal Knowledge Base Construction from Text-based Lifelogs. In Proceedings of SIGIR'19. 185-194.

[36] Saizheng Zhang, Emily Dinan, Jack Urbanek, Arthur Szlam, Douwe Kiela, and Jason Weston. 2018. Personalizing Dialogue Agents: I have a dog, do you have pets too?. In Proceedings of ACL'18.

[37] Yinhe Zheng, Guanyi Chen, Minlie Huang, Song Liu, and Xuan Zhu. 2019. Personalized Dialogue Generation with Diversified Traits. arXiv preprint arXiv:1901.09672 (2019) 